PRONOSTICO

\title{
Una regla de predicción clínica identifica pacientes con bajo riesgo de muerte por neumonía.
}

\begin{abstract}
Fine M, Thomas E, Auble Ph y col. New Engl J Med.1997; 336:243-50.

\section{Objetivo}

1)Identificar variables pronósticas en pacientes con neumonía de la comunidad para seleccionar aquellos con bajo riesgo de morir dentro de los 30 días.

2) Predecir días de internación, reinternaciones, y admisiones a terapia intensiva.
\end{abstract}

\section{Diseño}

Diseño y validación de una regla de predicción clínica.

\section{Lugar}

Base de datos de 275 hospitales de E.E.U.U.

\section{Pacientes}

Para el desarrollo de la regla se utilizaron datos de 14.199 pacientes hospitalizados por neumonía.

Se realizó la validación en dos grupos: a) 38.039 pacientes internados extraídos de una base de datos nacional; b) 2287 pacientes internados y ambulatorios de un estudio de cohortes de pacientes con neumonía (1). Se excluyeron pacientes menores de 18 años, pacientes HIV/Sida y pacientes internados durante los siete días previos o derivados de otra institución.

\section{Evaluación de los factores pronósticos}

Se evaluaron múltiples variables demográficas, enfermedades concomitantes, signos del examen físico y parámetros de laboratorio. La regla de predicción final incluyó sólo las variables asociadas significativamente a la muerte durante los treinta días subsiguientes.

\section{Medición de resultado principal}

El análisis de datos de 14199 pacientes internados con neumonía identificó variables que permitieron estratifi- carlos dentro de cinco clases de acuerdo al riesgo de muerte dentro de los 30 días.

\section{Resultados principales:}

La mortalidad a los 30 días fue baja para los pacientes de clase I $(0,1$ a $0,4 \%)$ II $(0,6$ a $0,7 \%)$, III $(0,9$ a $2,8 \%)$, y significativamente más alta en las clases IV $(8,5$ a $9,3 \%)$ y V $(27$ a $31 \%)$.

Pacientes de clase I $(9,7 \%)$ : < de 50 años; sin comorbilidades (cáncer, insuficiencia cardíaca, ACV, nefropatía o hepatopatía); sin alteraciones al examen físico (confusión, pulso $>125 / \mathrm{min}$., taquipnea $>30 / \mathrm{min}$, TA sistólica $<90 \mathrm{mmHg}$ o temperatura $<35^{\circ} \mathrm{C}$ o $40^{\circ} \mathrm{C}$ ).

Para clasificar a los pacientes de clase II a V se utilizó un sistema de puntuación en base a la presencia y relevancia de los siguientes factores:

\begin{tabular}{|lllc|}
\hline Edad (hombres) & Años & TA sist. $<90 \mathrm{mmHg}$ & +20 \\
Edad (mujeres) & Años -10 & Temp $<35 \mathbf{0}>\mathbf{4 0} \mathbf{~ C}$ & $\mathbf{+ 1 5}$ \\
Institucionalizado & +10 & Pulso $>125$ & +10 \\
Cáncer & +30 & $\mathrm{Ph}<7,35$ & +30 \\
Hepatopatía & +20 & Urea elevada & +20 \\
Insuf. Cardíaca & +10 & $\mathrm{Na}<130$ & +20 \\
ACV & +10 & Glucosa $>250 \mathrm{mg} / \mathrm{dl}$ & +10 \\
Nefropatía & +10 & Hto $<30 \%$ & +10 \\
Confusión & +20 & $\mathrm{~Pa} \mathrm{O} 2<60 \mathrm{mmHg}$ & +10 \\
Taquipnea $>30$ & +20 & Derrame pleural & +10 \\
\hline
\end{tabular}

Clase II: < 70 pts. Clase III: 71 a 90 pts Clase IV: 91 a 130 Pts. Clase V: $>130$ pts.

Hubo clara asociación entre la Clase y otros resultados analizados (Días de internación, UTI, y reinternaciones).

\section{Conclusiones:}

Esta regla de predicción clínica identifica con precisión a pacientes con neumonía de la comunidad con bajo riesgo de muerte y otros eventos adversos.

Apoyo económico: Agency for Health Care Policy and Research. EE.UU

\section{Comentario}

El trabajo constituye un modelo pronóstico a partir de reglas de predicción que ayuda a identificar a aquellos pacientes con neumonía que tienen bajo riesgo de morir dentro de los primeros 30 días de iniciada la enfermedad. Las reglas de predicción fueron derivadas y validadas con mas de 50000 pacientes internados en 275 hospitales. La validación prospectiva a través del estudio PORT (1) que incluye pacientes ambulatorios aumenta la validez externa. La regla de predicción clínica obtenida discriminó correctamente las subpoblaciones con distinto riesgo de muerte y otros eventos estudiados. La definición clara tanto de los criterios pronósticos como el resultado principal disminuyen los sesgos potenciales de los modelos de predicción. Ante una patología en la cual se observa una amplia variabilidad de conductas adoptadas por los médicos según la región, formación y lugar de trabajo, la aplicación de esta regla permitiría estratificar más objetivamente los riesgos y disminuir sustancialmente las internaciones innecesarias. Los autores sugieren cuidados ambulatorios para los pacientes de clase I y II; internación breve para clase III y internación tradicional para las clases IV y V. Como conclusión, el trabajo responde claramente al objetivo planteado y permite al médico ubicar al paciente con neumonía dentro de una determinada categoría de riesgo, lo que ayuda a determinar su conducta. Debemos enfatizar que las reglas de predicción clí- nica deben ser utilizadas como una herramienta más en el contexto del juicio clínico y nunca como una "receta de cocina".

\section{Dr. Martín Boccardo \\ Unidad de Medicina Familiar y Preventiva Hospital Italiano de Buenos Aires}

Referencias

1.Fine MJ, Hough Lj, Medsger AR et al. Hospital admission decision for patients with community acquired pneumonia: results from the Pneumonia PORT cohort study. Archives of Internal Medicine. (en impresión) 